\title{
Burnout estudiantil en universitarios veracruzanos
}

\section{Student Burnout in Veracruz University Students}

Burnout Estudante em universitário de Veracruz

DOI: http://dx.doi.org/10.23913/ride.v7i14.267

María Esther Barradas Alarcón

Facultad de Psicología, Universidad Veracruzana

ebarradas@uv.mx

Perfecto Gabriel Trujillo Castro

Instituto Tecnológico de Veracruz México. tecnito2009@prodigy.net.mx

Ana Virginia Sanchez Barradas

Facultad de Psicología, Universidad Veracruzana anita_07_sb@hotmail.com

Javier López González

Facultad de Psicología, Universidad Veracruzana

jalopez@uv.mx

\section{Resumen}

Introducción.- La formación académica del estudiante universitario, lo obliga a tener que enfrentar y resolver retos y desafíos del entorno, como son: horarios quebrados, manejo de tecnología, dominio de otro idioma, sobrecarga académica, tiempo limitado para estudiar para los exámenes que se les empalman, entrega de trabajos para el mismo día, el vivir solos, el no tener tiempo para alimentarse bien, el mal dormir, y la escases económica entre otros son fuente de estresores, a los que el estudiante está expuesto de manera permanente, facilitando con ello la posibilidad de presentar el síndrome de burnout estudiantil, que quiere decir, la presencia de un estado de agotamiento físico, emocional y cognitivo en el 
estudiante. Esta investigación tuvo como objetivo identificar el perfil descriptivo del síndrome de burnout estudiantil, que manifiestan los alumnos de la Licenciatura en Psicología y Administración de dos Universidades públicas en Veracruz, México. El Método fué cuantitativo y el nivel de estudio descriptivo. El Instrumento utilizado fue la Escala Unidimensional del Burnout Estudiantil. La población fue de 155 estuantes de Psicología de la Universidad Veracruzana y 101 de la Licenciatura en Administración del Instituto Tecnológico de Veracruz, un total de 256, en los Resultados, con una distribución del 100\% para cada carrera, se encontró con nivel alto de burnout estudiantil un $1.29 \%$ en mujeres de la licenciatura en administración, y un $0 \%$ en los hombres, en cuanto a la licenciatura en psicología ni hombres ni mujeres presentaron nivel alto de burnout estudiantil. Conclusiones. Se concluye que es necesario realizar investigaciones orientadas hacia la intervención, para erradicar la presencia de burnout estudiantil.

\section{Palabras claves: Nivel, burnout estudiantil}

\section{Abstract}

Introduction.- The academic education of University student, requires it having to confront and resolve challenges and challenges of the environment, such as: broken schedules, management of technology, proficiency in another language, academic overload, limited time to study for the exams that will abut them, works for same day delivery, living alone, not having time to feed is well, the bad sleep, and the scarcity economic among others are source of stressors, that the student is exposed of way permanent, facilitating with it the possibility of presenting the syndrome of student burnout, which means, the presence of a State of physical, emotional and cognitive depletion in the student. This research Objective is to identify the descriptive profile of the burnout syndrome student, showing the students of the degree in psychology and management of two public universities in Veracruz, Mexico. The method was quantitative and the level of study descriptive. The Instrument used was the One-dimensional Scale of the Student Burnout. The population was 155 students of Psychology of the University of Veracruz and 101 students of of Management from the Technological Institute of Veracruz, a total of 256, in the Results, with a distribution of $100 \%$ for each Bachelor's degree, met with high level of 
student burnout a $1.29 \%$ in women of the Bachelor's degree in Management, and $0 \%$ in men, with regard to the Bachelor's degree in psychology or men or women presented level high of burnout student. Conclusions. It is concluded that it is necessary to conduct the intervention-oriented research, to eradicate the presence of student burnout.

Key Words: Level, Student Burnout

\section{Resumo}

Introdutória estudante formação Academic College, obriga-o a ter de enfrentar e resolver desafios do ambiente, tais como: horários quebrados, tecnologia de manuseio, o domínio de outra língua, a sobrecarga acadêmica, tempo limitado para estudar para os exames são cabeçada, oferecendo trabalho para o mesmo dia, viver sozinho, não ter tempo para comer bem, falta de sono, e escassez económica entre outros, são uma fonte de estresse, que o aluno é exposto de forma permanente, facilitando, assim, a possibilidade de apresentar a síndrome de burnout estudante, o que significa a presença de um estado de exaustão física, emocional e cognitivo do aluno. Esta pesquisa teve como objetivo identificar o perfil descritivo da síndrome de burnout estudante, manifestando Grau estudantes de Psicologia e Administração de duas universidades públicas em Veracruz, México. O método foi o nível de estudo quantitativo e descritivo. $\mathrm{O}$ instrumento utilizado foi a escala unidimensional Burnout Student. A população era 155 estuantes de psicologia da Universidade Veracruzana e 101 da Licenciatura em Administração de Empresas pelo Instituto Tecnológico de Veracruz, um total de 256 nos resultados, com a distribuição de 100\% para cada corrida, ele encontrou alta esgotamento estudante 1,29\% em mulheres formado em administração, e $0 \%$ nos homens, quanto ao grau em psicologia nem os homens nem as mulheres tinham um alto nível de burnout aluno. Conclusões. Conclui-se que a investigação é necessária a intervenção orientada para erradicar a presença de burnout aluno.

Palavras-chave: Nível, estudante de burnout.

Fecha Recepción: Julio $2016 \quad$ Fecha Aceptación: Enero 2017 


\section{Introducción}

Hoy en día se vive una gran incertidumbre, ante la ola de violencia, inseguridad social, desempleo, inestabilidad económica y política, aunado a cambios vertiginosos en la ciencia, en la tecnología, a la globalización en general, que han afectado tanto a la naturaleza como al ser humano, pues han traído contextos sumamente complejos, que exigen a la persona mayor capacidad de adaptación, mayores recursos no solo psicológicos, también físicos.

Con frecuencia son tan altos los retos y las exigencias que no se puede con ellas, generándose un desequilibrio en la salud, como es el síndrome de burnout, definido como un síndrome de tres dimensiones: 1) Agotamiento o cansancio emocional, definido como el cansancio y fatiga que el trabajador experimenta, que puede manifestarse física y/o psíquicamente, con una sensación descrita de no poder dar más de sí mismo a los demás; 2) Despersonalización, definido como el desarrollo de sentimientos, actitudes y respuestas negativas distantes y frías hacia otras personas, especialmente hacia los beneficiarios del propio trabajo y por último; 3) Baja realización personal o logro, que se caracteriza por una dolorosa desilusión para dar sentido a la propia vida y hacia los logros personales, con sentimientos de fracaso y baja autoestima (Maslach y Jackson 1986).

Son alarmantes las cifras en torno a este problema, así la Organización Internacional del Trabajo (OIT), señala que el 75\% de los mexicanos que laboran presentan estrés laboral, porcentaje que pone a México entre los primeros lugares a nivel internacional, de ahí le sigue China con el 73\%, mientras que Estados Unidos presenta un 59\%, esta organización también comenta que esta enfermedad de estrés laboral no solo afecta al trabajador sino de manera directa a la economía de cualquier país, representando una perdida entre el $0.5 \%$ y $0.3 \%$ del Producto Interno Bruto (PIB) de cada nación. Por su parte el Instituto Nacional de Estadística y Geografía (INEGI, 2014), menciona que en México el PIB, cerro en este año con un incremento de $2.1 \%$, es decir las pérdidas económicas para la Nación son millonarias a causa del estrés laboral, sumado a lo anterior este padecimiento ha causado el $35 \%$ de los 75 mil infartos que año con año se registran en promedio en este país. El Instituto Mexicano del Seguro Social (IMSS) corrobora estas estadísticas señalando que de cada 10 trabajadores el 7.5 padece estrés laboral. 
La academia en la actualidad no escapa a esta situación y por lo tanto a la posibilidad de que el estrés y burnout estén presentes en quienes la integran, por lo que esta investigación tiene como propósito identificar el perfil descriptivo del síndrome de burnout estudiantil que manifiestan los alumnos de licenciatura de dos carreras en dos escuelas públicas.

Estrés Académico.- A partir de que Selye en 1960 publica que el estrés es "la suma de todos los efectos inespecíficos de factores (actividades cotidianas, agentes productores de enfermedades, drogas, hábitos de vida inadecuados, cambios abruptos en los entornos laboral y familiar), que pueden actuar sobre la persona" (Martínez y Díaz, 2007, p. 13).

Las diferentes disciplinas científicas como las ciencias médicas, químicas, biológicas y psicológicas se han esmerado en investigar desde su perspectiva el estrés. Es interesante señalar que hoy en día se estudia el estrés desde un enfoque integrador y multimodal, lo que significa que el estrés debe analizarse como un conjunto de variables que funcionan de forma sincrónica y diacrónica, dinamizadas a lo largo del ciclo vital.

Al desenvolverse el ser humano en tan diferentes contextos, es posible que en cada uno de ellos se presente el estrés, de acuerdo a esto, Barraza (2005, p. 3) señala que "los tipos de estrés suelen ser clasificados tomando como base la fuente del estrés (...), en ese sentido se puede hablar del estrés amoroso y marital, del estrés sexual, del estrés familiar, del estrés por duelo, del estrés médico, del estrés ocupacional, del estrés académico, del estrés militar y del estrés por tortura y encarcelamiento". Luego entonces el estrés generado en un ambiente académico se le llama estrés académico, estrés escolar, estrés de examen, entre otros.

Lamentablemente la escuela es considerada como una fuente de estrés para todos los que en ella se encuentran, tenemos por ejemplo que la docencia es considera como una de las profesiones más estresantes, al respecto Feitler y Tolkar (1997), muestran que un 16 por ciento de los catedráticos consideran su profesión como "muy estresante" o "extremadamente estresante". Continuando con los catedráticos, en un estudio realizado por Barradas (2015) con una población de 24 profesores del área de ciencias de la salud y 30 profesores del área de humanidades, con rangos de edad entre los 30 a 70 años y con grado educativo de maestría y doctorado, utilizando como instrumento el test Perfil de Estrés de Novak, encontró que el $4.16 \%$ manifiestan un nivel de estrés que representa un riesgo para 
la salud, mientras que el $29.16 \%$ presentan relativa fortaleza ante situaciones de estrés y sus efectos a la salud.

En cuanto al otro sector de la comunidad académica; los estudiantes, esta misma autora señala que la evaluación del maestro, la sobrecarga de trabajo, las exposiciones en el aula, la elaboración de ensayos o trabajos de investigación, y la tensión con sus profesores, son factores considerados por los propios estudiantes, como estresores que favorecen, el que vivan situaciones que los obligan a generar malos hábitos alimenticios, tener problemas de sueño o no dormir lo suficiente por tener que estudiar y realizar tareas, también favorece el que consuman de manera regular drogas, como el alcohol u otro tipo de droga. En este sector académico se tienen algunos estudios como el de: Bittar (2008) que señala que 56.9\% de los estudiantes de posgrado de Salud Pública de la Universidad de Guadalajara presentan burnout. O el estudio de Dyrbye y Shanafelt (2002) quienes afirman que el 45\% de los estudiantes de los últimos años, de tres facultades de medicina, de la Clínica de Minnesota presentan el síndrome de burnout.

Consecuencias del burnout.- El burnout genera consecuencias tanto personales, familiares como laborales, según señalan los estudios más relevantes de la literatura médica sobre el tema. Sin embargo, aparecen numerosas discrepancias entre diversos autores respecto a cuáles son las principales consecuencias que afectan a los docentes en su ámbito de trabajo, algunos mencionan que en el ámbito personal entre las consecuencias destacan la incapacidad para desconectarse del trabajo, los problemas de sueño, el cansancio que aumenta la susceptibilidad a la enfermedad, problemas gastrointestinales, de espalda, cuello, dolores de cabeza, enfermedades coronarias, sudor frío, náuseas, taquicardia, aumento de enfermedades virales y respiratorias relacionadas en numerosas ocasiones con el abuso de fármacos tranquilizantes, estimulantes, café, tabaco, alcohol y otras drogas, destacando en la bibliografía la proliferación en las cifras de consumo de alcohol entre los profesores.

En la misma línea, algunos otros autores encuentran que mayores puntuaciones en despersonalización dan lugar a un incremento de los trastornos psicofisiológicos, mientras que otros señalan que un alto nivel de estrés predice problemas de salud física y mental. 
Asimismo, Matud, M.P., García, M.A. y Matud, M.J. (2002) señalan que la insatisfacción con el rol laboral y la presión en el trabajo se correlacionan con sintomatología de tipo somático, depresiva, de ansiedad e insomnio.

Por otro lado, Calvete y Villa (2000) presentan coeficientes de correlación significativos entre el cansancio emocional y síntomas de depresión, somatización, ansiedad, dificultades cognitivas y sensibilidad interpersonal. Estos mismos señalaron que las consecuencias que afectan a nivel psicológico al profesor que padece burnout son numerosas: falta de autorrealización, baja autoestima, aislamiento, tendencia a la autoculpa, actitudes negativas hacia uno mismo y hacia los demás, sentimientos de inferioridad y de incompetencia, pérdida de ideales, irritabilidad, hasta casos más extremos de intentos de suicidio, cuadros depresivos graves, ansiedad generalizada, fobia social y agorafobia.

Finalmente, cabe señalar como consecuencia evidente del estrés y el síndrome de burnout, la insatisfacción laboral y/o académica, citada por un alto número de autores.

\section{Método}

Tipo de investigación. El estudio es de tipo descriptivo que de acuerdo a Hernández, Fernández y Baptista (2014), pretende establecer las características, los niveles de asociación, las formas de manifestación o los índices de ocurrencia del problema de investigación.

Método. Se realizó a través de una metodología cuantitativa; que según Hernández, Fernández y Baptista (2014) es el método que utiliza la recolección y el análisis de datos para contestar preguntas de investigación, confiando en la medición numérica, el conteo y frecuentemente en el uso de la estadística para establecer con exactitud patrones de comportamiento en una población.

Definición de variable.- Burnout.- Estado de agotamiento físico, emocional y cognitivo producido por el involucramiento prolongado en situaciones generadoras de estrés. (Barraza, A. 2008). 
Instrumento.- La Escala Unidimensional del Burnout Estudiantil (EUBE). El instrumento está constituido por 15 preguntas que pueden ser respondidas mediante la escala tipo Likert de cuatro valores asignados (nunca, algunas veces, casi siempre y siempre). Tiene una confiabilidad de 0.91 y un alfa de Cronbach de 0.89 en la confiabilidad por mitades; estos resultados han sido valorados como muy buenos de acuerdo a la escala de valoración asignada por De Vellis (Barraza 2008).

Objetivo de Investigación.- identificar el perfil descriptivo del síndrome de burnout que manifiestan de la licenciatura en Administración del Instituto Tecnológico de Veracruz y la Licenciatura en Psicología de la Universidad Veracruzana.

Población.- 256 estudiantes de los cuales 155 son de la Facultad de Psicología de la Universidad Veracruzana de ellos 48 son hombres y 107 son mujeres. Y 101 alumnos de la Licenciatura en Administración del Instituto Tecnológico de Veracruz de los cuales 24 son hombres y 77 son mujeres. Ver. Tabla 1 
Tabla 1 Características sociodemográficas de los estudiantes participantes.

\begin{tabular}{|c|c|c|c|}
\hline CATEGORÍA & CARACTERÍSTICA & $\mathbf{N}$ & $\%$ \\
\hline \multicolumn{4}{|l|}{ Facultad } \\
\hline & Psicología UV & 155 & 60.5 \\
\hline & Administración ITV & 101 & 39.4 \\
\hline \multicolumn{4}{|l|}{ Sexo } \\
\hline & Hombre & 72 & 28.1 \\
\hline & Mujer & 184 & 71.8 \\
\hline \multicolumn{4}{|l|}{ Edad (media) } \\
\hline & 20.6 años de edad & & \\
\hline \multicolumn{4}{|l|}{ Estado Civil } \\
\hline & Soltero & 245 & 95.7 \\
\hline & Casado & 7 & 2.7 \\
\hline & Otro & 4 & 1.5 \\
\hline \multicolumn{4}{|l|}{ No. De Hijos } \\
\hline & $\mathrm{Si}$ & 10 & 3.9 \\
\hline & No & 246 & 96.0 \\
\hline \multicolumn{4}{|l|}{ trabaja } \\
\hline & $\mathrm{Si}$ & 60 & 23.4 \\
\hline & No & 196 & 76.5 \\
\hline \multicolumn{4}{|l|}{ Generación } \\
\hline & Iniciales & 107 & 41.7 \\
\hline & Intermedios & 36 & 14.0 \\
\hline & Avanzados & 113 & 44.1 \\
\hline
\end{tabular}

Fuente: Propia 


\section{Resultados}

\section{Figura 1}

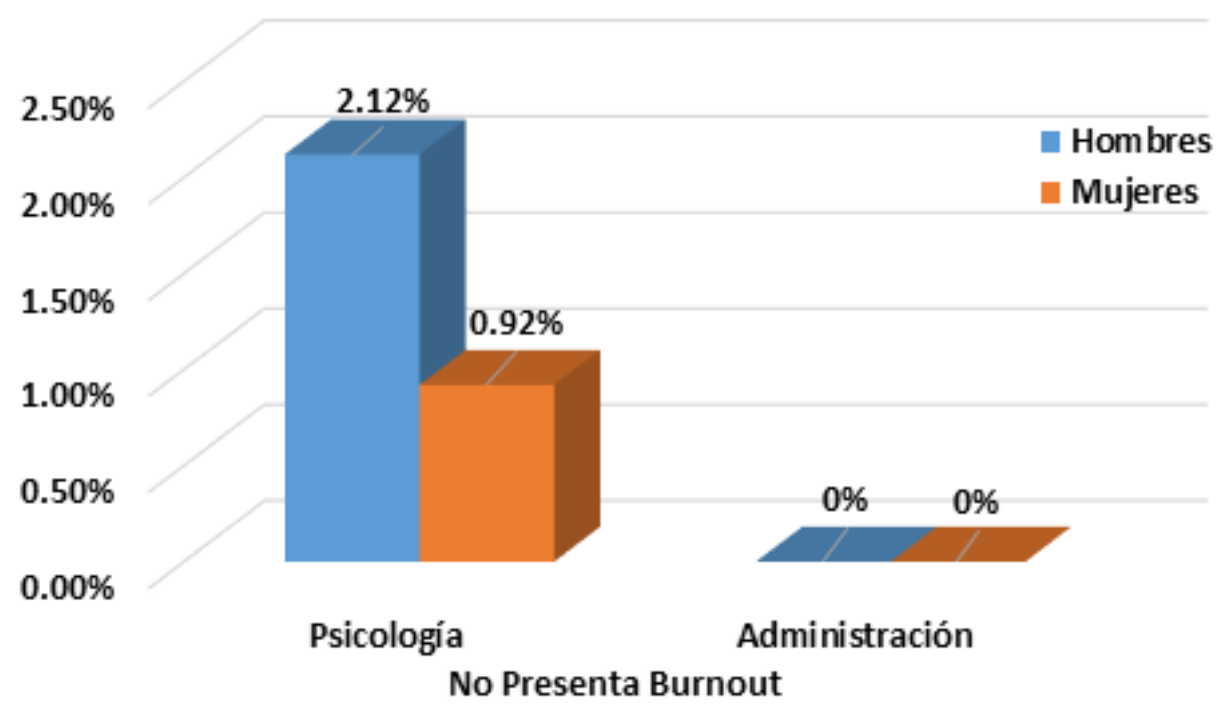

Figura 1. De acuerdo al sexo no se presentó Burnout Estudiantil en alumnos de Lic. en Psicología y Lic. en Administración

Figura 2

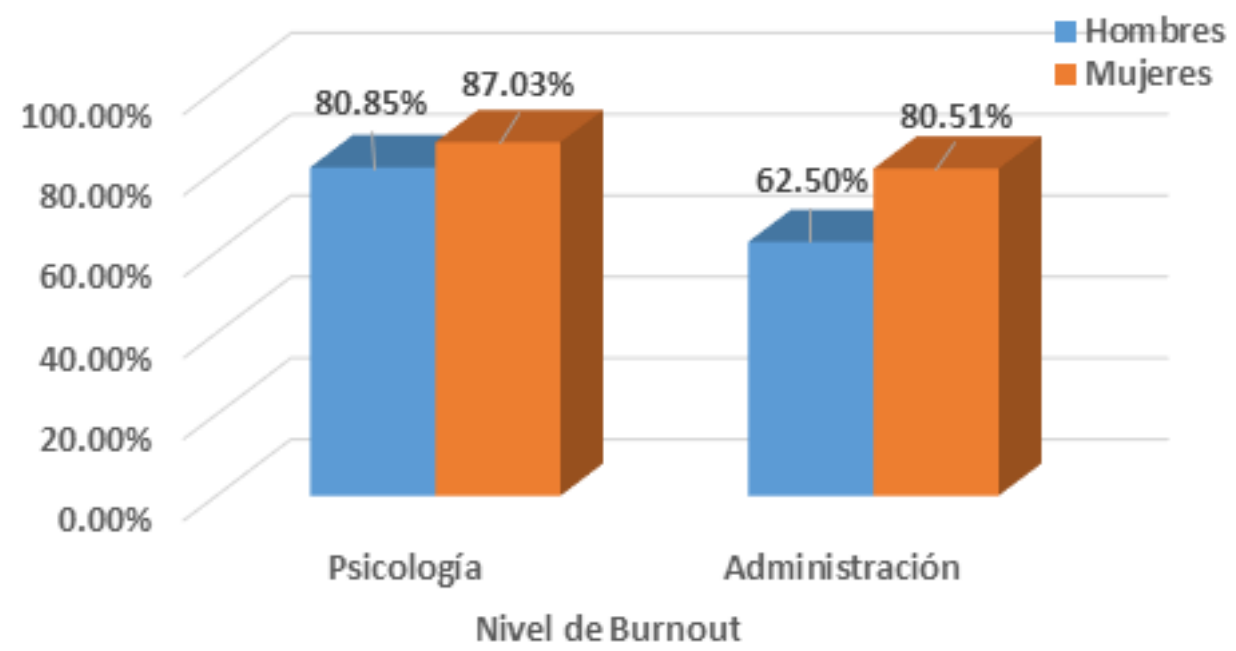


Figura 2. De acuerdo al sexo Nivel Leve de Burnout Estudiantil en alumnos de Lic. en Psicología y Lic. en Administración

\section{Figura 3}

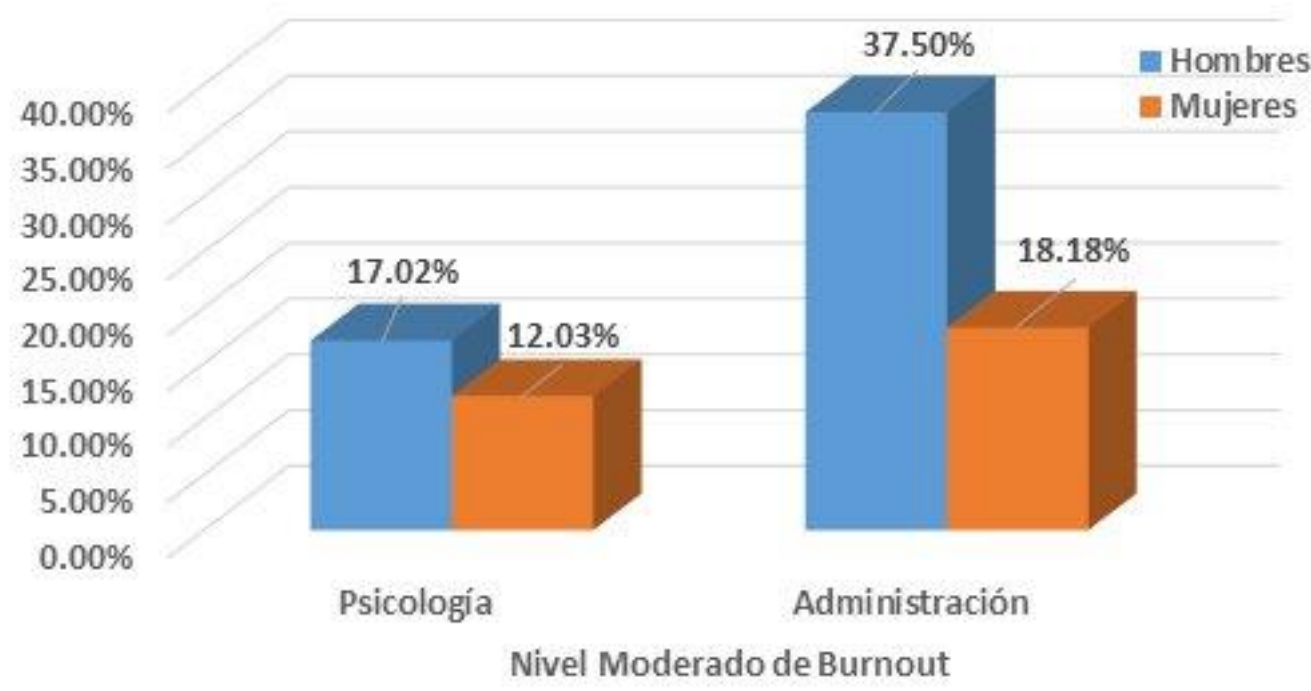

Figura 3 De acuerdo al sexo Nivel Leve de Burnout Estudiantil en alumnos de Lic. en Psicología y Lic. en Administración

\section{Figura 4}

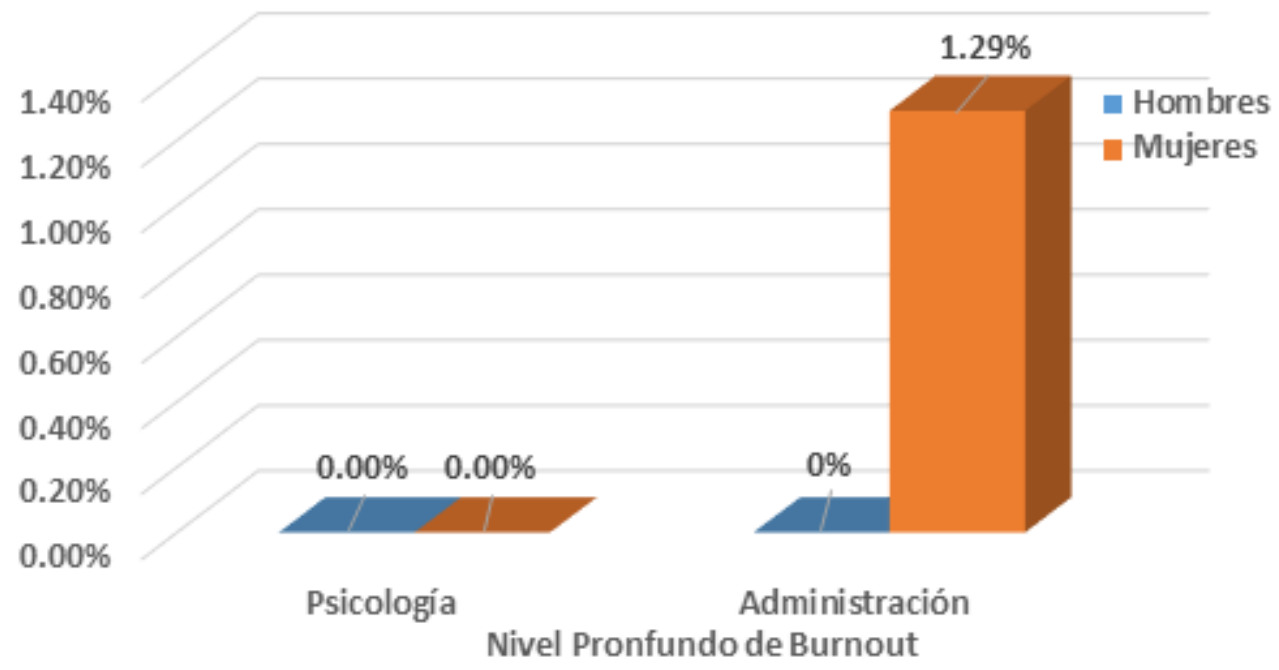


Figura 4. De acuerdo al sexo Nivel Leve de Burnout Estudiantil en alumnos de Lic. en Psicología y Lic. en Administración

\section{Figura 5}

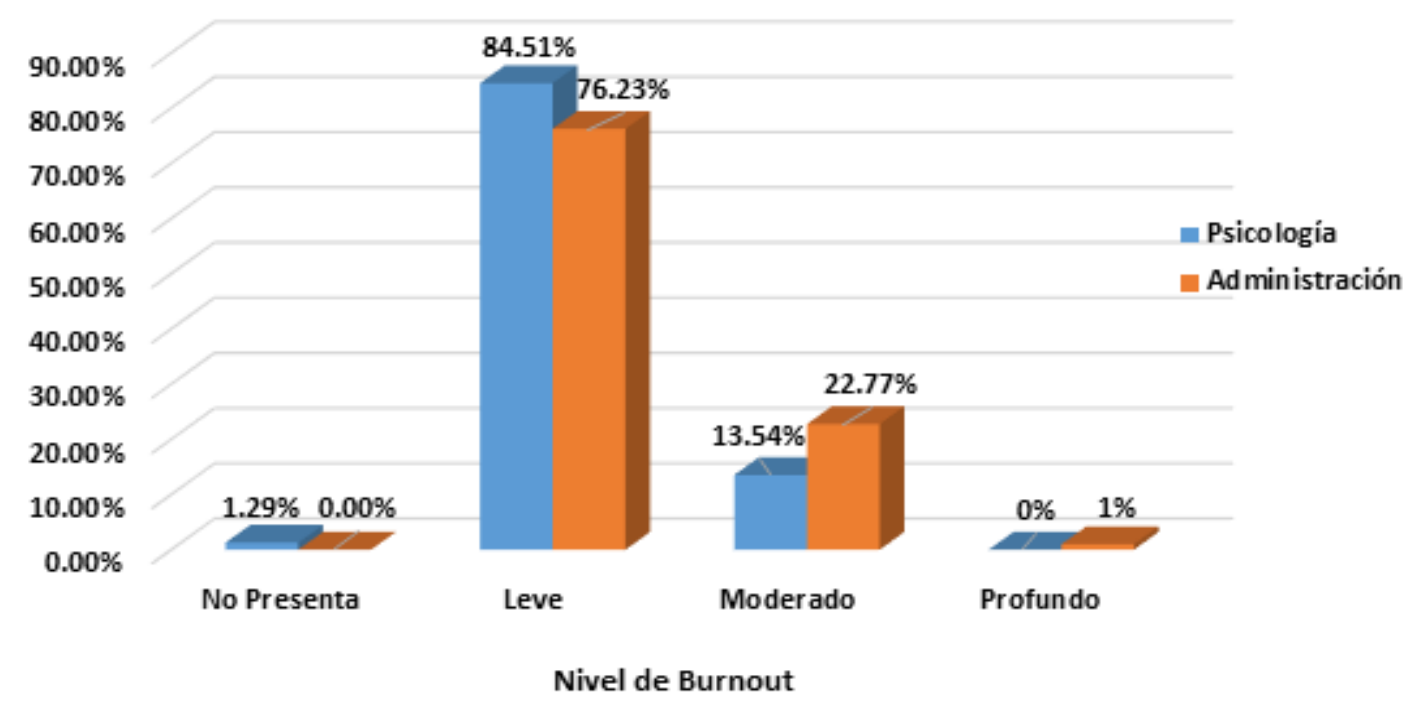

Fig

ura 5. Con población general en cada carrera, los Niveles de Burnout Estudiantil en alumnos de Lic. en Psicología y Lic. en Administración

\section{Figura 6}

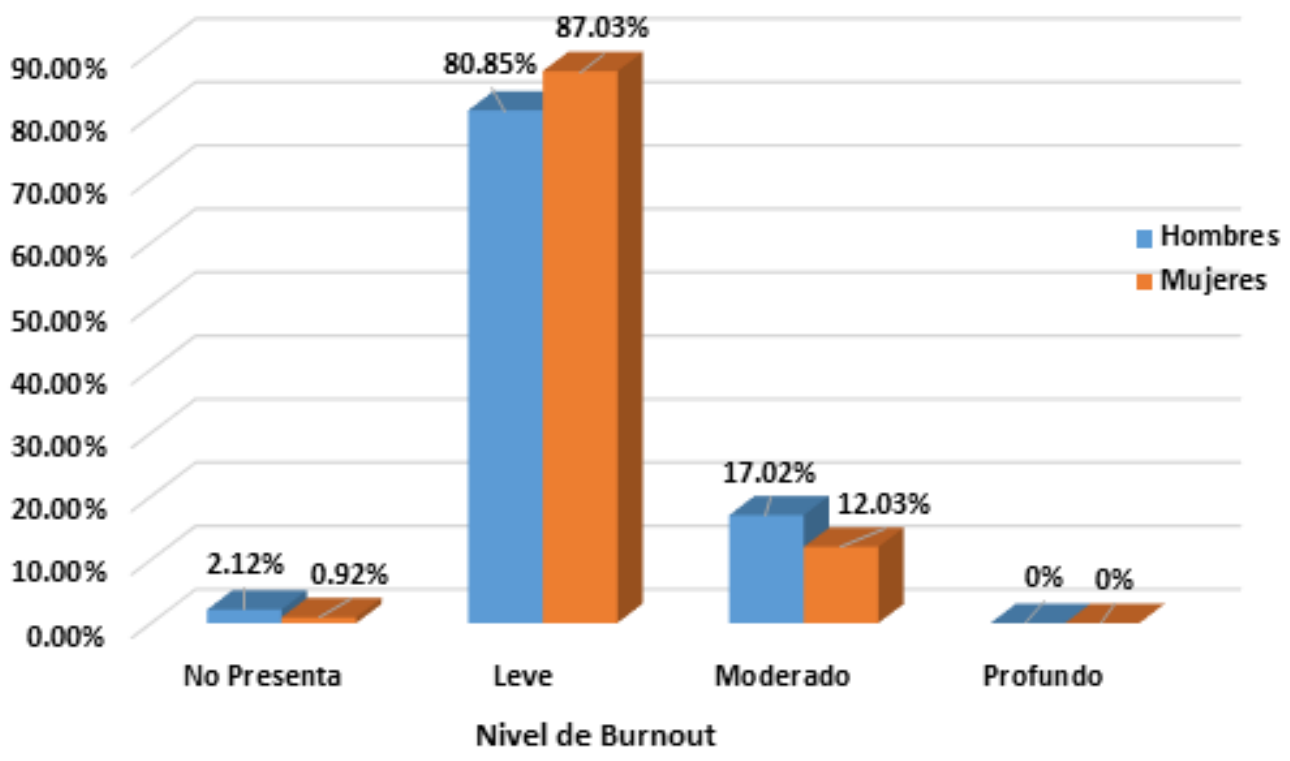

Vol. 7, Núm. 14

Enero - Junio $2017 \quad$ RIDE 
Figura 6. De acuerdo al sexo Niveles de Burnout Estudiantil en alumnos de Lic. en Psicología de la Universidad Veracruzana

\section{Figura 7}

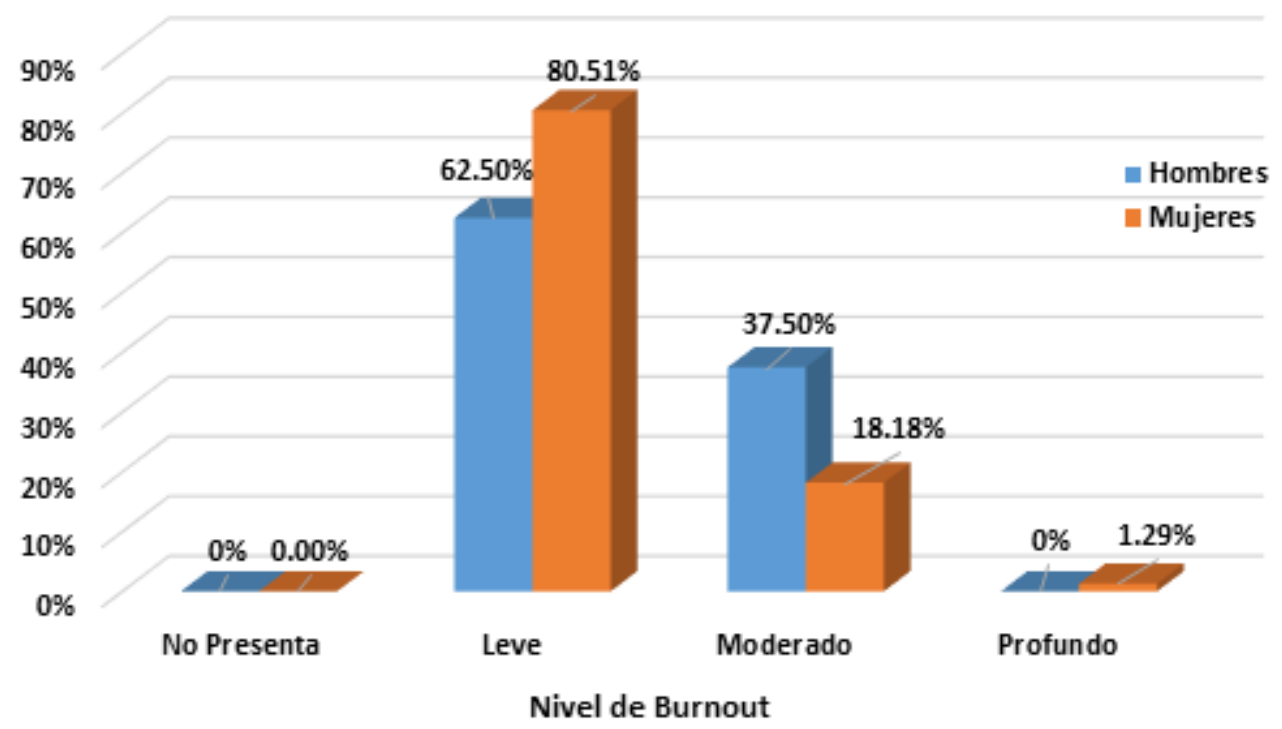

Figura 7. De acuerdo al sexo Niveles de Burnout Estudiantil en alumnos de Lic. en Administración del Instituto Tecnológico de Veracruz

\section{Discusión}

El presente trabajo aborda el tema del burnout no tan estudiado en el ámbito académico y sobre todo en estudiantes. El burnout es considerado como el resultado de exponerse de manera prolongada en situaciones generadoras de estrés hasta llegar al extremo de no tomar en cuenta las necesidades personales, generando con ello un estado de agotamiento físico, emocional y cognitivo (Barraza 2009). El objetivo planteado fue identificar el nivel del síndrome de burnout en estudiantes de la Licenciatura en Psicología de la Universidad Veracruzana $(n=155)$ y estudiantes la licenciatura en Administración del Instituto Tecnológico de Veracruz $(n=101)$. Se utilizo la Escala Unidimensional del Burnout Estudiantil. Los resultados encontrados revelan la existencia de burnout estudiantil, en mayor porcentaje en nivel leve, siguiendo el nivel moderado y solo en una carrera el nivel 
profundo, estos son los primeros hallazgos en ambas carreras sobre el burnout estudiantil, siendo este uno de los aportes principales de la investigación, lo que favorece ratificar el modelo sistémico cognoscitivista del estrés académico (Barraza, 2007ª , Berrio y Mazo, 2011), que señala la interacción de las variables del estrés, como filtro a través del cual se valoran los estímulos estresores que rodean al estudiante es decir esas representaciones internas de las evaluaciones particulares y problemáticas. Resultados similares a los encontrados en este estudio fueron los señalados por Barraza (2009) quien utilizando la misma escala Unidimensional del Burnout Estudiantil en una población total de 663 estudiantes de dos carreras en las que se confirma que alumnos encuestados presentan un nivel leve de este síndrome.

En la presente investigación se observó que en ambas carreras son los estudiantes hombres quienes presentan burnout leve, así como en ambas carreras, son las mujeres con porcentajes mayores de burnout moderado. Parecidos a estos resultados fueron los encontrados por Moreno-Jiménez B, Carvajal R.R., Escobar R.E. (2001) en una muestra de 51 alumnos de la Facultad de Enfermería y Obstetricia de la Universidad Juárez del Estado de Durango, a los cuales se les aplicó la Escala Unidimensional de Burnout Estudiantil encontraron que todos los alumnos encuestados presentaron el síndrome de burnout, destacando éste nivel leve ( $84 \%$ de los encuestados).

En otro estudio en esta ocasión realizado por Rosales R. (2012), con 70 estudiantes de primer año en la Universidad de Ciencias Médicas de Holguín, Cuba; 35 de cada sexo, a quienes se les aplicó el mismo instrumento Escala Unidimensional de Burnout Estudiantil, se encontró que la mayoría de ellos manifestaron el síndrome de burnout leve, y fue el sexo femenino el más afectado.

Así mismo los resultados encontrados por Caballero et al. (2007) fueron nuevamente similares a los antes señalados, ellos trabajaron con estudiantes de psicología en los que encontró, que un $41.6 \%$ de ellos presentaron el síndrome de burnout estudiantil, además se describían así mismos con la sensación de no poder dar más de ellos mismos y una actitud cínica sobre el valor y sentido de sus tareas como estudiantes. El 38.2\% experimentó agotamiento, el $29.7 \%$, cinismo y el $48.6 \%$, un sentimiento de ineficacia. Hay otro estudio de la autora Bittar (2008), que señala resultados parecidos a los encontrados en este 
estudio y en el de los otros autores, con respecto a los estudiantes de psicología ella señala que el $56.9 \%$ de los estudiantes de posgrado de salud pública de la Universidad de Guadalajara presentan burnout. Según esta investigadora, el síndrome se distribuyó de acuerdo a la carrera de la siguiente manera: el 34\% estudiantes de Derecho, $13 \%$ estudiantes de Psicología, 13\% de Administración, 10\% de Comercialización, 9\% de Contaduría pública, 8\% de Relaciones internacionales, 6\% de Sistema de información, 3\% de Turismo, y $2 \%$ de Comunicación.

Será interesante observar si el género es importante para que estos resultados se presenten. Dentro de las fortalezas de este estudio pueden señalarse: la representatividad del grado académico, las características de las dos instituciones públicas de educación superior, el mismo instrumento utilizado, al igual que la participación voluntaria de los estudiantes. También se presentan limitaciones: el número de participantes, ya que solo representa la generación particular de cada carrera en ambas instituciones educativas, además la ausencia de otros instrumentos que nos permitieran ver los síntomas que podían ya estar presentado los participantes de este estudio, así como las características sociales, económicas y culturales que no fueron consideradas para el mismo.

\section{Conclusiones}

Como en su momento se señaló, existen resultados de investigación en los que se demuestra cómo es que el ámbito académico es considerado como fuente de estrés para todos los que en ella se encuentran, así tenemos que el estudiante se enfrenta a una serie de estresores tales como la sobrecarga académica, trabajos obligatorios, participación ante el grupo y masificación (hacinamiento en el aula), y particularmente en el de la Universidad Veracruzana y el Instituto Tecnológico de Veracruz, su modelo educativo genera tensión pues no siempre se ofertan las experiencias educativas que requieren para ir sumando su avance crediticio, o los horarios son demasiados quebrados dejando muchas horas libres entre una clase y otra, lo que genera que no puedan asistir a sus casa para comer, o no tengan tiempo suficiente para dormir bien. En ocasiones la oferta y demanda de las experiencias educativas se saturan y ya no pueden elegir las que requieren, por otro lado, la economía de la mayoría de estos estudiantes en ambas instituciones es muy escasa, situación que les genera tensión al tener que enfrentar gastos en materiales académicos, 
varios de ellos se tienen que ayudar llevando algo que vender en la escuela, estas son algunas de las situaciones difíciles a las que se enfrentan los estudiantes. Considerando entonces que el involucramiento prolongado a situaciones generadoras de estrés como las arriba señaladas, llega a producir burnout, indica que el estudiante expuesto a estas situaciones de estrés de manera permanente, llega a experimentar estado de agotamiento físico, emocional y cognitivo, razones suficientes que motivaron la presente investigación en la que se encontró lo siguiente:

Comparando por cada nivel de burnout estudiantil con una ponderación del cien por ciento para hombres y cien por ciento para las mujeres en cada carrera:

En el Nivel leve de burnout estudiantil se encontró en Psicología un $80 \%$ de hombres y un $87.0 \%$ en mujeres, y en Administración un $62.5 \%$ de hombres y un $80.5 \%$ en mujeres. .

En cuanto al Nivel moderado de burnout estudiantil en Psicología se encontró un 17.0\% en hombres y un $12.0 \%$ en mujeres, y en Administración un 37.5\% en hombres y un $18.1 \%$ en mujeres.

En cuanto al Nivel profundo de burnout estudiantil en Psicología no se encontraron casos, mientras que en Administración se encontró el 1.29\% en mujeres.

Por otro lado tomando en consideración una ponderación del $100 \%$ para el total de población, en cada carrera tenemos que:

El 84.5\% de estudiantes en general de Psicología y el 76.23\% de estudiantes en general de Administración, presentan un nivel leve de burnout. Mientras que el 13.54\% de estudiantes de psicología y el $22.77 \%$ de estudiantes de Administración presentan un nivel moderado de burnout. Y solo el $1.29 \%$ de estudiantes de Administración presentan un nivel profundo de burnout.

Y finalmente comparando los niveles de burnout al interior de cada carrera con una ponderación del $100 \%$ para cada sexo tenemos que:

El nivel de burnout estudiantil que sobresale en la Facultad de Psicología es el nivel leve con el $80.8 \%$ en hombres y el $87.0 \%$ en mujeres, mientras que el nivel moderado de 
burnout estudiantil se encontró un $17.02 \%$ en hombres y un $12.0 \%$ en mujeres. Y no se reportó el nivel profundo de burnout estudiantil en ningun sexo en esta licenciatura.

En la licenciatura en Administración también sobresale el nivel leve de burnout con el $62.5 \%$ en hombres y el $80.5 \%$ en mujeres, seguido de un nivel moderado de burnout estudiantil con el $37.5 \%$ en hombres y el $18.18 \%$ en mujeres. Si se encontró el nivel profundo de burnout estudiantil solo en mujeres con el $1.29 \%$.

Lo anterior nos obliga a sugerir para próximos estudios, tomar en consideración otras variables, como son las demográficas, se sugiere tener una muestra más grande y representativa de diferentes carreras y si es posible con modelos educativos diferentes. Así mismo generar investigación de intervención fundamentada en el modelo sistémico cognoscitivista del estrés académico, con el fin de habilitar a los estudiantes en diferentes técnicas de afrontamiento, para que puedan dar un adecuado manejo a los estímulos estresores a los que está expuesto. 


\section{Bibliografía}

APA, American Psychological Association. (1998). Trastornos Adaptativos. En DSM IV. Manual diagnóstico y estadístico de los Trastornos Mentales. Barcelona. Masson, 1995: 639-43. En José Luis Vázquez-Barquero (ed), Psiquiatría en Atención Primaria. Grupo Aula Médica, España.

Barradas A., Trujillo C., Guzmán I., López G., Leo A. (2015, 8 de julio) Nivel de estrés en docentes universitarios. Revista Iberoamericana de Contaduría, Economía y Administración.

https://www.ricea.org.mx/index.php/ricea/article/view/38

Barraza, A. (2008, 21 de noviembre). Burnout estudiantil: un enfoque unidimensional. Revista PsicologiaCientifica.com, 10 (30).

http://www.psicologiacientifica.com/burnout-estudiantil

Barraza, A. (2005, 29 marzo). Características del estrés académico de los alumnos de educación media superior. PsicologíaCientífica.com, 19 (1).

https://dialnet.unirioja.es/descarga/articulo/2880918.pdf

Barraza, A. (2009, 30 noviembre). Estrés Académico y Burnout Estudiantil. análisis de su relación en alumnos de licenciatura. Psicogente 12 (22): pp. 272-283.

http://dialnet.unirioja.es/descarga/articulo/3265008.pdf

Bittar C. (2008, 7 julio). Burnout y estilos de personalidad en estudiantes universitarios. Cátedra Iberoamericana.

http://fci.uib.es/Servicios/dif_invest/Difunda-su-investigacion.cid221990

Caballero, C., Abello, R. \& Palacio, J. (2007 marzo). Relación del burnout y rendimiento académico con la satisfacción frente a los estudios en estudiantes universitarios. Avances en Psicología Latinoamericana, 25 (2).

https://revistas.urosario.edu.co/index.php/apl/article/view/1208

Calvete, E. y Villa, A. (2000, junio.). Burnout y síntomas psicológicos: modelo de medida y relaciones estructurales. Revista Ansiedad y Estrés 6, 117-130.

http://pendientedemigracion.ucm.es/info/seas/Revista/list_crono.htm

Dyrbye, L. \& Shanafelt, T. (2002, junio). Estudiantes de medicina y médicos quemados. http://www.intramed.net/contenidover.asp?contenidoID $=41127$ 
Feitler y Tolkar (1997), citado por Travers, Ch. J. \& Cooper, L. (1997). El estrés de los profesores: La presión en la actividad docente. Barcelona: Paidós.

Matud, M.P., García, M.A. y Matud, M.J. (2002 septiembre). Estrés laboral y salud en el profesorado: un análisis diferencial en función del género y del tipo de enseñanza. International Journal of Clinical and Health Psychology, 2, 451-465.

http://www.aepc.es/ijchp/articulos_pdf/ijchp-50.pdf

Hernández, R.; Fernández, C. y Baptista, P. (2014). Metodología de la investigación. México: Editorial McGraw-Hill.

Maslach, C. y Jackson, S.E. (1986). MBI (2 Ed., $1^{\circ}$ Ed. De 1981). Palo Alto, California: Consulting Psychologist Press.

Maslach, C. \& Jackson, S. (1981, junio). The measurement of experienced burnout. Journal of Social Issues.

http://psychsource.bps.org.uk/details/journalArticle/3432521/The-measurement-ofexperienced-burnout.html

Moreno González A. (2009, junio). Síndrome de Burnout en estudiantes de educación física y deportes de la universidad de Tolima. Revista EDU-Física, 2 (6).

\section{http://edu-fisica.com/Revista 2/SINDROME DE BURNOUT.pdf}

Moreno Jiménez, B., Carvajal, R. R. \& Escobar, R. E. (2001, junio). La evaluación del burnout profesional. Factorialización del MBI-GS. Un análisis preliminar. Revista Ansiedad y Estrés, 7 (1), 69-78.

Martínez Díaz, E. S. \& Díaz Gómez, D. A. (2007, Noviembre). Una aproximación psicosocial al estrés escolar. Educación y Educadores, 10(2) pp. 11-22.

http://www.redalyc.org/pdf/834/83410203.pdf

Martínez Díaz ES, y Díaz Gómez DA. (2007 diciembre) Una aproximación psicosocial al estrés escolar. Educ Educadores.

http://disde.minedu.gob.pe/bitstream/handle/123456789/2209/2007_Martínez_Una aproximación psicosocial al estrés escolar.pdf

Rosales Ricardo, Y. (2012, junio) Estudio unidimensional del síndrome de burnout en estudiantes de medicina de Holguín. Revista de la Asociación Española de $\begin{array}{llll}\text { Neuropsiquiatría, } & 32 & \text { (116), }\end{array}$ http://www.ww.revistaaen.es/index.php/aen/article/view/16574 